\title{
Severe blunt trauma in Finland and Estonia: comparison of two regional trauma repositories
}

\author{
Sten Saar ${ }^{1,2} \cdot$ Tuomas Brinck $^{3} \cdot$ Juhan Laos ${ }^{1,2} \cdot$ Lauri Handolin $^{3} \cdot$ Peep Talving ${ }^{1,2}$
}

Received: 29 July 2018 / Accepted: 26 December 2018 / Published online: 5 January 2019

(c) Springer-Verlag GmbH Germany, part of Springer Nature 2019

\begin{abstract}
Purpose Evolving trauma system of Estonia has undergone several reforms; however, performance and outcome indicators have not been benchmarked previously. Thus, we initiated a baseline study to compare demographics, management and outcomes of severely injured patients between Southern Finland and Northern Estonia utilizing regional trauma repositories. Methods A comparison of data fields of the Helsinki University Hospital trauma registry (HTR) and trauma registry at the North Estonia Medical Centre in Tallinn (TTR) between 1/1/2015 and 31/12/2016 was performed. The inclusion criterion was Injury Severity Score $>15$. Transferred patients, patients with penetrating injuries, and pediatric patients were excluded. The data for comparison included demographics, Trauma Score-Injury Severity Score (TRISS), mortality, and standardized mortality ratio (SMR). Primary outcome was mortality and SMR per TRISS methodology.

Results During the 2-year study period, 324 patients from the HTR and 152 from the TTR were included. Demographic profile was similar between the repositories with the exception of severe abdominal injuries being more prevalent at the TTR $(25.0 \%$ vs. $13.3 \%, p=0.002)$. Predominant injury mechanism was non-ground level fall in both repositories. Mortality was similar at $14.5 \%$ and $13.6 \%$ at the TTR and HTR, respectively (adj. $p=0.762$; OR 1.13, 95\% CI 0.64-1.99). SMR was lower at the HTR compared to the TTR ( 0.65 vs. $0.77, p>0.05)$, however, the difference did not reach statistical significance. Conclusion Benchmarking trauma repositories at a national level provides opportunities for quality and performance improvements. We observed comparable demographic profile and outcome indicators in the compared regional trauma systems.
\end{abstract}

Keywords Severe trauma $\cdot$ Trauma registry $\cdot$ Comparison

\section{Introduction}

It is estimated globally that after every $6 \mathrm{~s}$ someone deceases secondary to an injury [1]. Trauma repositories are important tools to monitor quality indicators and outcomes in trauma systems, regions and trauma facilities [2, 3].

The Helsinki University Hospital (HUH) trauma center is the regional blunt trauma receiving facility in the Southern

Peep Talving

peep.talving@ut.ee

1 School of Medicine, Institute of Clinical Medicine, University of Tartu, Tartu, Estonia

2 Division of Acute Care Surgery, Department of Surgery, North Estonia Medical Centre, J. Sütiste tee 19, 13149 Tallinn, Estonia

3 Trauma Unit, Department of Orthopaedics and Traumatology, Töölö Hospital, Helsinki University Hospital and University of Helsinki, Helsinki, Finland
Finland. The catchment area of HUH is up to $34,378 \mathrm{~km}^{2}$ with a population of 2 million inhabitants. In the Northern Estonia region, all severely injured patients independent of trauma mechanism are admitted to the North Estonia Medical Centre (NEMC) trauma center in Tallinn with estimated population of 800,000 inhabitants in the catchment area of $24,597 \mathrm{~km}^{2}$.

The Helsinki University Hospital's (HUH) trauma registry (Helsinki Trauma Registry, HTR) was established in 2005 and has been benchmarked previously against UK trauma facilities utilizing Trauma Audit and Research Network (TARN) and German level-one trauma centers using TraumaRegister $\mathrm{DGU}^{\circledR}$ repository $[4,5]$. A trauma repository at the NEMC in Tallinn (TTR) covering the Northern Estonia was developed in 2015 and no previous benchmarking with established trauma repositories in the region has been conducted. Thus, the aim of the current investigation was to benchmark demographics, management and outcomes of severely injured blunt trauma victims of the 
Northern Estonia trauma system against the Southern Finland, utilizing regional trauma repositories. This investigation serves as a baseline study for the evolving Estonian regional trauma system.

\section{Materials and methods}

\section{Study populations}

A comparison of case records at the HTR and TTR between $1 / 1 / 2015$ and 31/12/2016 was performed. The inclusion criterion was Injury Severity Score (ISS) $>15$ [6, 7]. Transfers from outside hospitals, patients $<16$ years and patients sustaining penetrating trauma were excluded to increase the comparability of data as patients with penetrating truncal trauma and patients less than 16 years of age are transported to another affiliated trauma facility in the Southern Finland trauma system.

\section{Trauma registries in Northern Estonia and Southern Finland}

Both registries follow the Utstein criteria and the data input is conducted by trained nurses. The inclusion criteria for TTR is ISS $>15$ and for HTR New Injury Severity Score (NISS) $>15$ of patients treated at the resuscitation room, however, only patients with ISS $>15$ were included in the current study.

\section{Data comparison and statistics}

The data comparisons included demographics, injury characteristics, mechanism of injury, pre-hospital data, emergency department (ED) investigations, hospital length of stay (HLOS), intensive care unit length of stay (ICU-LOS), TRISS score (Trauma Score-Injury Severity Score), 30-day observed and adjusted in-hospital mortality, and standardized mortality ratio (SMR; ratio of observed deaths to predicted deaths) [8]. A Glasgow Coma Scale (GCS) score registered at ED on admission was utilized for analysis, however, if GCS score on admission was missing or the patient was intubated prior to arrival at hospital the first on-scene GCS was included. The TRISS score was calculated using the Revised Trauma Score (RTS) including systolic blood pressure, respiratory rate and GCS score on admission, however, if the patient was intubated or RTS on admission was missing an on-scene RTS was utilized. Ventilator days were defined as mechanical ventilator time but continuous positive airway pressure (CPAP) days were not counted.

Primary outcome was 30-day in-hospital mortality and SMR per the TRISS score.

Secondary outcomes were HLOS and ICU-LOS.
The $p$ values for continuous variables were derived from the Student $t$ test or Mann-Whitney tests and for categorical values Chi square or 2-sided Fisher's test were deployed. Logistic regression analysis was used to compare the adjusted mortality between the regions. $p$ values $<0.05$ were considered statistically significant. Values are reported as mean \pm standard deviation $(\mathrm{SD})$ for continuous variables and as a percentage for categorical variables. Logistic regression analysis was used to compare mortality adjusted for significantly different $(p<0.05)$ demographic variables and mechanism of injuries. All statistical analyses were performed with the R-program version 3.3.2 (R Foundation, Vienna, Austria).

\section{Results}

During the 2-year study period, 324 patients from the HTR and 152 from the TTR were included for the final analysis (Fig. 1).

Demographics and clinical characteristics of all patients are depicted in Table 1. A significantly higher proportion of patients with severe abdominal injuries were admitted to the $\operatorname{NEMC}(p=0.002)$.

The rate of transferred patients was significantly higher at the HUH $(p<0.001)$ (Fig. 1).

Mechanism of injuries is listed in Table 2. The predominating mechanism of injury was non-ground level fall in both trauma systems. Prevalence of bicycle injuries was more frequent in Southern Finland $(p=0.008)$.

Pre-hospital data and ED investigations are depicted in Table 3. The rate of pre-hospital intubation was significantly higher in Southern Finland $(p<0.001)$. One out of 20 patients were transported to the HUH with a helicopter, however, ground transportation only was utilized in Northern Estonia. The use of the Focused Assessment with Sonography in Trauma (FAST) was significantly higher at the NEMC and inversely a chest X-ray (CXR) was performed more frequently at the HUH. Computed tomography (CT) utilization was equally deployed in both trauma systems.

Interventional radiology was used in $4.6 \%$ and $3.7 \%$ of the patients at the NEMC and the HUH, respectively $(p=0.828)$ (Table 3).

HLOS and ICU-LOS was significantly longer at the NEMC, however, ventilator days were similar at the compared systems (Table 4).

The crude 30-day in-hospital mortality was similar at $14.5 \%$ and $13.6 \%$ at the NEMC and HUH, respectively $(p=0.904)$. Multivariate mortality comparison between trauma systems adjusted for AIS abdomen $\geq 3$, non-ground level falls and bicycle injuries resulted in an odds ratio of 1.13 with a confidence interval of $0.64-1.99(p=0.762)$ (Table 4). TRISS score was 81.1 in Northern Estonia and 79.2 in Southern Finland. 


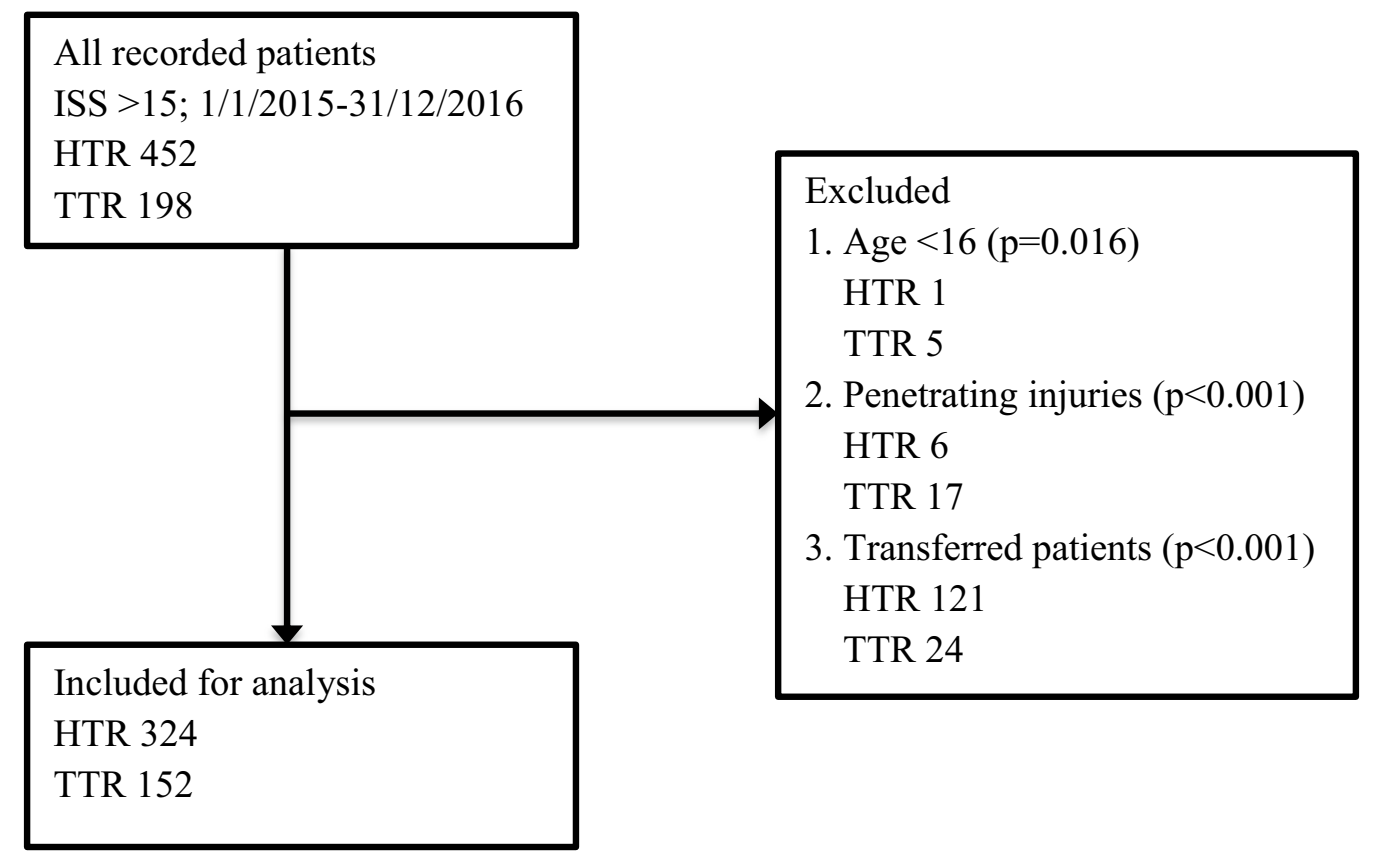

Fig. 1 Flowchart of all included patients. ISS Injury Severity Score, HTR Helsinki Trauma Registry, TTR Tallinn Trauma registry

Table 1 Demographics and clinical injury characteristics

\begin{tabular}{llll}
\hline$n$ & TTR & HTR & $p$ value \\
& 152 & 324 & \\
\hline Age (years) & $47.7 \pm 19.2$ & $48.4 \pm 20.1$ & 0.694 \\
Age $>65$ years & $19.7 \%(30)$ & $23.5 \%(76)$ & 0.429 \\
Male & $73.7 \%(112)$ & $72.5 \%(235)$ & 0.878 \\
GCS $<9$ & $28.3 \%(43)$ & $33.6 \%(109)$ & 0.288 \\
SBP $<90 \mathrm{mmHg}$ & $12.5 \%(19)$ & $9.0 \%(29)$ & 0.300 \\
ISS & $25.7 \pm 10.1$ & $26.1 \pm 9.7$ & 0.678 \\
AIS head $\geq 3$ & $56.6 \%(86)$ & $61.4 \%(199)$ & 0.366 \\
AIS chest $\geq 3$ & $52.0 \%(79)$ & $60.2 \%(195)$ & 0.112 \\
AIS abdomen $\geq 3$ & $25.0 \%(38)$ & $13.3 \%(43)$ & $\mathbf{0 . 0 0 2}$ \\
AIS extremity $\geq 3$ & $27.6 \%(42)$ & $31.5 \%(102)$ & 0.456 \\
\hline
\end{tabular}

Statistically significant value is in bold

HTR Helsinki Trauma Registry, TTR Tallinn Trauma registry, GCS Glasgow Coma Scale, SBP systolic blood pressure, ISS Injury Severity Score, AIS Abbreviated Injury Scale

Standardized mortality ratio (SMR) was lower at the HUH, however, did not reach statistical significance (Table 4).

Most patients treated at NEMC were discharged home (50.7\%), while most patients $(58.0 \%)$ treated at HUH were discharged to step-down hospitals (Table 4).
Table 2 Mechanism of injury

\begin{tabular}{llll}
\hline$n$ & TTR & HTR & $p$ value \\
& 152 & 324 & \\
\hline Motor vehicle accident & $17.1 \%(26)$ & $15.1 \%(49)$ & 0.676 \\
Auto vs. pedestrian & $11.2 \%(17)$ & $5.9 \%(19)$ & 0.063 \\
Motorcycle accident & $8.6 \%(13)$ & $12.0 \%(39)$ & 0.328 \\
Bicycle accident & $3.9 \%(6)$ & $12.0 \%(39)$ & $\mathbf{0 . 0 0 8}$ \\
Ground level fall & $11.8 \%(18)$ & $15.1 \%(49)$ & 0.413 \\
Non-ground level fall & $32.9 \%(50)$ & $23.8 \%(77)$ & $\mathbf{0 . 0 4 6}$ \\
Other or unknown & $14.5 \%(22)$ & $16.0 \%(52)$ & 0.759 \\
\hline
\end{tabular}

Statistically significant values are in bold

HTR Helsinki Trauma Registry, TTR Tallinn Trauma Registry

\section{Discussion}

The current study is a pioneering investigation validating the evolving Estonian trauma system against established Finnish regional trauma system performance using regional trauma repositories. The trauma system in Southern Finland with a relatively long existing trauma registry and a comparable injury profile serves as an excellent baseline study for the Estonian trauma system.

The overall demographic profile of the two cohorts was similar with a sole exception of a higher admission rate of severe abdominal injuries at the Estonian system that may result from greater proportion of non-ground level falls resulting in higher energy transfer and more frequent 
Table 3 Pre-hospital times, intubation, helicopter transportation and emergency department investigations

\begin{tabular}{lllr}
\hline$n$ & $\begin{array}{l}\text { TTR } \\
152\end{array}$ & $\begin{array}{l}\text { HTR } \\
324\end{array}$ & $p$ value \\
\hline Overall pre-hospital time (min) & $56 \pm 30$ & $78 \pm 70$ & $<\mathbf{0 . 0 0 1}$ \\
Pre-hospital intubation (all patients) & $13.2 \%$ & $37.3 \%$ & $<\mathbf{0 . 0 0 1}$ \\
Pre-hospital intubation (GCS 3-8) & $44.2 \%$ & $87.2 \%$ & $<\mathbf{0 . 0 0 1}$ \\
ED intubation (all patients) & $22.4 \%$ & $9.6 \%$ & $<\mathbf{0 . 0 0 1}$ \\
ED intubation (GCS 3-8) & $55.1 \%$ & $4.6 \%$ & $<\mathbf{0 . 0 0 1}$ \\
Helicopter transportation & 0 & $4.7 \%$ & $\mathbf{0 . 0 0 4}$ \\
CXR & $32.2 \%$ & $50.0 \%$ & $<\mathbf{0 . 0 0 1}$ \\
FAST & $93.4 \%$ & $73.5 \%$ & $<\mathbf{0 . 0 0 1}$ \\
CT & $95.4 \%$ & $94.4 \%$ & 0.831 \\
Interventional radiology & $4.6 \%$ & $3.7 \%$ & 0.828 \\
\hline
\end{tabular}

Statistically significant values are in bold

HTR Helsinki Trauma Registry, TTR Tallinn Trauma Registry, GCS Glasgow Coma Scale, ED emergency department, $C X R$ chest X-ray, $F A S T$ focused assessment with sonography in trauma, $C T$ computed tomography

abdominal injuries. The higher proportion of transferred patients in the Southern Finland can be explained by the larger catchment area of the Helsinki University Hospital with a need of temporary stabilization at local hospitals. The mean age of patients was similar despite higher average life expectancy in Finland compared to Estonia (81.1 vs. 77.6 years) [9].
Non-ground level falls and GLF contribute to the most of the cases in both regions and similar results have been reported by other authors from European trauma centers [10, 11]. GLF as a trauma mechanism may appear "insignificant", however, frequently resulting in serious injuries especially in the head and cervical region $[12,13]$. A study by Velmahos et al. reported that during a 9-month study period $37 \%$ of patients admitted to a Level I trauma center after GLF or low-level fall had significant injuries often requiring ICU admission [12]. An independent risk factor for significant injuries was older age, thus, both our study regions are at a "risk group" with a rather high mean age and significant proportion of GLF-particularly in Southern Finland with almost quarter of the patients being older than 65 years. Also, frequent use of anticoagulants among elderly population exposes to a higher risk for hemorrhage.

Severe bicycle injuries are uncommon in the Estonian population and may be affected by a low popularity of bicycles as an everyday transportation vehicle in Estonia compared to Finland. Similar to Finland, a recent investigation from the Netherlands reported bicycle injuries as a second most common mechanism of injury reflecting the high prevalence of bicycle use [14].

Pre-hospital intubation of injured patients is a controversial subject with rather weak evidence base. A recent study by Haltmeier et al. investigating patients with severe isolated head injury and field GCS $<9$ reported significantly higher mortality for patients with pre-hospital endotracheal
Table 4 Hospital length of stay, TRISS, 30-day mortality and discharge destination (transferred patients excluded)

\begin{tabular}{llll}
\hline$n$ & TTR & HTR & $p$ value \\
& 152 & 324 & \\
\hline HLOS (days) & $26.3 \pm 36.1$ & $12.4 \pm 10.3$ & $\mathbf{0 . 0 0 3}$ \\
ICU-LOS (days) (if treated in ICU) & $14.3 \pm 17.5$ & $8.5 \pm 7.3$ & $\mathbf{0 . 0 4 4}$ \\
Ventilation days (if ventilated) & $10.7 \pm 15.3$ & $6.6 \pm 6.3$ & 0.288 \\
TRISS & $81.1 \pm 26.2$ & $79.2 \pm 25.5$ & \\
Expected mortality & $18.9 \%$ & $20.8 \%$ & \\
30-day in-hospital crude mortality & $14.5 \%$ & $13.6 \%$ & 0.904 \\
30-day adjusted mortality & & & $0.762($ OR \\
& & & $0.13 ; 95 \%$ CI \\
& & & $>0.05$ \\
Difference $^{\text {c }}$ (SMR & d & & $-7.99)$ \\
Step-down hospital $_{\text {Rehabilitation }}$ & $-4.4 \%(0.77)$ & $58.0 \%$ & $<\mathbf{0 . 0 0 1}$ \\
Home $^{\text {Other/unknown }}$ & $18.1 \%$ & $6.2 \%$ & $<\mathbf{0 . 0 0 1}$ \\
\hline
\end{tabular}

Statistically significant values are in bold

HTR Helsinki Trauma Registry, TTR Tallinn Trauma Registry, HLOS hospital length of stay, ICU-LOS intensive care unit length of stay, SMR standard mortality ratio

${ }^{\mathrm{a}} 100.0 \%$-TRISS

${ }^{\mathrm{b}}$ Adjusted for AIS abdomen $\geq 3$, bicycle accident, non-ground level falls

c30-day crude (observed) mortality-expected mortality

d30-day crude (observed) mortality/expected mortality 
intubation compared to patients without pre-hospital intubation performed [15]. A study by Bossers and colleagues observed that outcome of pre-hospital intubation depends on the skills and experience of the pre-hospital providers and should be abandoned when a provider lacks ample training [16]. In the current study, the pre-hospital intubation rate was notably higher in the Finnish trauma system. The causality for this observation is unclear and may be related to greater transportation distances at the catchment area of the HUH and more frequent presence of a physician on the scene. However, as TTR does not register if the on-scene care provider was a paramedic or physician, a detailed analysis is not feasible, thus, this serves as a quality improvement purpose for the TTR to add a data field to record if a paramedic or physician was on-scene.

None of the severely injured patients in Estonia were transported by helicopter compared to almost 5\% helicopter transportations in Southern Finland. However, the subject is still controversial and a recent Cochrane Database metaanalysis could not find clear benefits of helicopter transportation due to lack of high-quality evidence and heterogeneity of the studies [17]. Nevertheless, there may be a potential benefit for certain subgroups of patients transported from highways distant to the hospital or during rush hours, potentially decreasing pre-hospital times [18].

Utilization of CT-imaging exceeded $90 \%$ at both hospitals and may be related to a relatively low number of hemodynamically unstable patients on admission providing time for precise and time-consuming diagnostics.

HLOS was remarkably higher at the NEMC and the most obvious explanation is significantly higher use of step-down hospitals as a discharge destination at the $\mathrm{HUH}$.

The previous study comparing HTR with the DGU ${ }^{\circledR}$ registry in Germany reported that HLOS and ICU-LOS have remained identical at the $\mathrm{HUH}$, however, HLOS and ICU-LOS at the NEMC was similar to the data of Germany hospitals [5]. Per the DGU ${ }^{\circledR}$ registry, mean HLOS and ICULOS was 25 and 12 days compared to 26 and 14 days at the NEMC, respectively [5]. Thus, the differences in HLOS between Southern Finland and Northern Estonia are affected by the local policies. One possible solution to decrease HLOS at the NEMC could be the increased use of stepdown hospitals, which may be also cost-effective, however, this needs political decisions at a national level in Estonia.

Crude and adjusted mortality were comparable between the regions. Also, both regions showed better outcome compared to predicted mortality per TRISS methodology, however, due to relatively low number of cases the SMR should be interpreted with caution. TRISS score is a widely used tool to monitor the performance of trauma centers, however, with many well-known limitations. One of the most important limitations is simplistic dichotomous stratification of age variable: $\leq 55$ and $>55$ years. With increasing average of life-expectancy and evolving medical care make such a strict cut-off per age questionable. Also, some authors have shown that TRISS performance is low for traumatic brain injuries and for certain mechanisms of injuries. Kennedy and co-authors noted that TRISS methodology has a poor performance for patients after low-level falls [19]. Patients in this subgroup have frequently many chronic medical conditions with an effect on outcomes.

Trauma courses to improve outcome of severely injured patients have been recently introduced in both compared trauma systems: European Trauma Course (ETC) in Finland in 2011, Advanced Trauma Life Support (ATLS) in Estonia in 2015, Definitive Surgical Trauma Care (DSTC) and Definitive Anesthetetic Trauma Care (DATC) courses and Advanced Surgical Skills for Exposure in Trauma (ASSET) in 2017 and 2018 in both countries, respectively [20-23]. Different from Finland, the ATLS course is mandatory for emergency medicine, orthopedics, general surgery, pediatric surgery, radiology and anesthesiology and intensive care residency programs in Estonia since 2017. These educational instruments may have a significant effect for outcomes following implementations, thus, it would be of interest to commence a new comparison between the registries after a few years.

The current investigation suffers limitation inherent to a relatively short study period and a low number of cases, however, as TTR was developed recently, it was not feasible to perform a comparison involving a longer time periods. Also, the TRISS methodology has many limitations, however, with wide utilization it permits comparison across different trauma registries. Nevertheless, we think this study is important for baseline and validation of TTR and for overall trauma system in Estonia to improve outcomes and initiate new studies in the future.

\section{Conclusion}

Benchmarking trauma repositories at a national level provides opportunities for quality and performance improvements. We observed comparable demographic profile and outcome indicators in the compared regional trauma systems.

Funding None.

\section{Compliance with ethical standards}

Conflict of interest Sten Saar, Tuomas Brinck, Juhan Laos, Lauri Handolin, and Peep Talving declare that they have no conflict of interest.

Ethical approval All procedures performed in studies involving human participants were in accordance with the ethical standards of the insti- 
tutional and/or national research committee and with the 1964 Helsinki declaration and its later amendments or comparable ethical standards.

\section{References}

1. World Health Organization (WHO). Injuries and violence. The Facts. 2014. http://apps.who.int/iris/bitstream/10665/14979 8/1/9789241508018_eng.pdf?ua $=1 \& u a=1 \& u a=1$. Accessed 29 Jan 2018

2. Moore L, Clark DE. The value of trauma registries. Injury. 2008;39(6):686-95.

3. Lefering R, Ruchholtz S. Trauma registries in Europe. Eur J Trauma Emerg Surg. 2012;38(1):1-2.

4. Handolin L, Leppäniemi A, Lecky F, Bouamra O, Hienonen P, Tirkkonen S, Pihlström K, Yates D, Hirvensalo E. Helsinki Trauma Outcome Study 2005: audit on outcome in trauma management in adult patients in southern part of Finland. Eur J Trauma Emerg Surg. 2008;34(6):570-6.

5. Brinck T, Handolin L, Paffrath T, Lefering R. Trauma registry comparison: six-year results in trauma care in Southern Finland and Germany. Eur J Trauma Emerg Surg. 2015;41(5):509-16.

6. Baker SP, O'Neill B, Haddon W Jr, Long WB. The injury severity score: a method for describing patients with multiple injuries and evaluating emergency care. J Trauma. 1974;14(3):187-96.

7. Gennarelli TA, Wodzin E, et al. The Abbreviated Injury Scale 2005. Update 2008. Des Plaines: American Association for Automotive Medicine (AAAM); 2008.

8. Boyd CR, Tolson MA, Copes WS. Evaluating trauma care: the TRISS method. Trauma Score and the Injury Severity Score. J Trauma. 1987;27(4):370-8.

9. World Health Rankings. http://www.worldlifeexpectancy.com/ world-health-rankings. Accessed $24 \mathrm{Feb} 2018$.

10. Harmsen AMK, Giannakopoulos GF, Terra M, de Lange de Klerk ESM, Bloemers FW. Ten year maturation period in a levelI trauma center, a cohort comparison study. Eur J Trauma Emerg Surg. 2017;43(5):685-90.

11. Bilén K, Ponzer S, Castrén M, Pettersson H, Ottosson C. The impact of trauma mechanism on outcome: a follow-up study on health-related quality of life after major trauma. Eur J Trauma Emerg Surg. 2010;36(5):449-55.
12. Velmahos GC, Jindal A, Chan LS, Murray JA, Vassiliu P, Berne TV, Asensio J, Demetriades D. "Insignificant" mechanism of injury: not to be taken lightly. J Am Coll Surg. 2001;192(2):147-52.

13. Helling TS, Watkins M, Evans LL, Nelson PW, Shook JW, Van Way CW. Low falls: an underappreciated mechanism of injury. J Trauma. 1999;46(3):453-6.

14. El Mestoui Z, Jalalzadeh H, Giannakopoulos GF, Zuidema WP. Incidence and etiology of mortality in polytrauma patients in a Dutch level I trauma center. Eur J Emerg Med. 2017;24(1):49-54.

15. Haltmeier T, Benjamin E, Siboni S, Dilektasli E, Inaba K, Demetriades D. Prehospital intubation for isolated severe blunt traumatic brain injury: worse outcomes and higher mortality. Eur J Trauma Emerg Surg. 2017;43(6):731-9.

16. Bossers SM, Schwarte LA, Loer SA, Twisk JW, Boer C, Schober $\mathrm{P}$. Experience in prehospital endotracheal intubation significantly influences mortality of patients with severe traumatic brain injury: a systematic review and meta-analysis. PLoS One. 2015;10(10):e0141034.

17. Galvagno SM Jr, Sikorski R, Hirshon JM, Floccare D, Stephens C, Beecher D, Thomas S. Helicopter emergency medical services for adults with major trauma. Cochrane Database Syst Rev. 2015;15(12):CD009228.

18. Talving P, Teixeira PG, Barmparas G, DuBose J, Inaba K, Lam L, Demetriades D. Helicopter evacuation of trauma victims in Los Angeles: does it improve survival? World J Surg. 2009;33(11):2469-76.

19. Kennedy RL, Grant PT, Blackwell D. Low-impact falls: demands on a system of trauma management, prediction of outcome, and influence of comorbidities. J Trauma. 2001;51(4):717-24.

20. European Trauma Course. http://www.europeantraumacourse .com. Accessed 10 Apr 2018.

21. The American College of Surgeons. Advanced trauma life support. https://www.facs.org/quality-programs/trauma/atls. Accessed 3 Mar 2018

22. International Association for Trauma Surgery and Intensive care. Definitive surgical trauma care. http://www.iatsic.org/DSTC/. Accessed 3 Mar 2018.

23. The American College of Surgeons. Advanced surgical skills for exposure in trauma. https://www.facs.org/quality-programs/traum a/education/asset. Accessed 3 Mar 2018. 